\title{
DIAGNÓSTICO ANATOMOHISTOPATOLÓGICO DE PLATINOSOMOSE EM FELINO: RELATO DE CASO
}

\author{
Tayane Kelly de CARVALHO ${ }^{*}$, Lilian Cristina de Sousa Oliveira BATISTA ${ }^{1}$, Luiz Augusto Laureano \\ SAMPAIO $^{2}$ \& Ana Paula ARAGÃO ${ }^{1}$
}

1 Faculdade de Medicina Veterinária de Valença. Valença, Rio de Janeiro, Brasil.

2 Médico Veterinário Autônomo. Barra do Piraí, Rio de Janeiro, Brasil.

*Autor para correspondência: tayane_kc@ hotmail.com

DOI: http://dx.doi.org/10.18571/acbm.149

\section{RESUMO}

A platinosomose é uma parasitose de grande relevância na medicina felina, causada pelo parasito Platynosomum spp. O ciclo de vida deste parasito não é completamente conhecido por sua complexidade, o gato é o hospedeiro definitivo que elimina ovos nas fezes. Ao infectar-se ocorrem obstrução e inflamação dos ductos biliares, além do acometimento de outros locais. Os sinais clínicos são inespecíficos dificultando seu diagnóstico definitivo. O objetivo do presente trabalho foi relatar um caso de platinosomose diagnosticado post mortem, através de estudos anatomohistopatológicos de um felino doméstico adulto, fêmea, castrada, com aproximadamente seis anos de idade, atendida na Policlínica Veterinária Escola da Faculdade de Medicina Veterinária de Valença (FMVV), Rio de Janeiro.

Palavras chave: Icterícia; Fígado; Platynosomum spp.

\begin{abstract}
Anatomohistopathological diagnosis of platinosomosis in feline: Case Report. Platinosomosis is a parasite of great relevance in the feline medicine, caused by the parasite of the genus Platynosomum spp. The life cycle of this parasite is not completely known by its complexity, the cat is the definitive host that eliminates eggs in the feces. Upon infection, there is obstruction and inflammation of the bile ducts, in addition to the involvement of other sites. The symptomatology is nonspecific, making its definitive diagnosis difficult. The objective of the present study was to report a case of platinosomosis diagnosed post mortem, through anatomohistopathological studies of an adult female, female, castrated feline with approximately six years of age, attended at the Polyclinic Veterinary School of the Faculty of Veterinary Medicine of Valença (FMVV), Rio de Janeiro.
\end{abstract}

Keywords: Jaundice; Liver; Platynosomum spp.

\section{Introdução}

Platinosomose é o nome dado a doença causada pelo parasitismo por Platynosomum spp., que é uma das principais hepatopatias que acometem os felinos (AZEVEDO et al., 2013). Após a lipidose hepática que é bastante comum, as colangites e colangiohepatites são classificadas como a segunda causa de doença hepática mais comum nos gatos (SOLDAN; MARQUES, 2011).

$\mathrm{Na}$ infecção pelo Platynosomum spp. ocorrem obstrução e inflamação dos dutos biliares (LEAL et al., 2011). Causando manifestações clínicas como inapetência, letargia, anemia, icterícia, 


\section{ACTA

diarreia mucóide, vômito, ascite, hepatomegalia (MICHAELSEN et al., 2012). A semelhança da sintomatologia da platinosomose com as de outras patologias, ou mesmo a falta de sinais podem passar despercebidos para a parasitose, dificultando assim o controle e tratamento da mesma (FILHO et al., 2015).

A complexidade do ciclo de vida do parasita dificulta que este seja totalmente conhecido, sabese que inclui até três hospedeiros intermediários (CARREIRA et al., 2008). Nas regiões com tipo de clima quente, os gatos infectam-se ingerindo, por exemplo, lagartixas e sapos, pelo hábito predatório (FILHO et al., 2015).

A forma de evitar essa parasitose, seria evitar o contato dos gatos com os hospedeiros intermediários, mas é uma situação complicada de se fazer, já que os felinos têm o hábito de predatório (PAULA, 2010). A única solução possível, segundo Franco et al. (2013), depende dos médicos veterinários diagnosticarem a parasitose precocemente para que o tratamento instituído tenha eficácia e facilite recuperação do animal acometido. Para tal diagnóstico precoce seria necessário que se tornasse rotina a realização de exames parasitológicos de fezes dos animais (PAULA, 2010).

O objetivo do trabalho é relatar um caso de platinosomose em gato diagnosticada em exame post mortem.

\section{Relato de Caso}

O presente trabalho relata um caso de um felino doméstico adulto, sem raça definida, fêmea, castrada, com aproximadamente seis anos de idade, atendida na Policlínica Veterinária Escola da Faculdade de Medicina Veterinária de Valença (FMVV), Rio de Janeiro. Na realização da anamnese o proprietário relatou que a gata apresentava um comportamento letárgico, andava pelo quintal da residência prostrada, sem interesse por alimentos há cerca de cinco dias, mesmo com o oferecimento de ração associada a outros alimentos.

No exame físico revelou uma ligeira icterícia, suspeitando-se inicialmente de intoxicação. $\mathrm{O}$ animal foi submetido à fluidoterapia com cloreto de sódio a $0,9 \%$ combinado a um estimulante energético desintoxicante (Stimovit@) e vitamina B12; e posteriormente uma sonda nasogástrica foi inserida para administração de dieta. Visto que o animal precisava de acompanhamento médico, permaneceu internado na localidade durante o dia, retornando à noite para casa. $\mathrm{O}$ animal não apresentou melhoras significativas com o tratamento instituído.

Ao retornar a Policlínica, dias depois, o felino que continuava sem se alimentar e ictérico, agora apresentava desidratação severa. Foi solicitada a realização de exames complementares como hemograma completo, pesquisa de hematozoários, bioquímica sérica e ultrassonografia. O resultado do hemograma demonstrou que os parâmetros estavam dentro da normalidade. A pesquisa de hematozoários revelou resultado negativo. Já na bioquímica sérica observou-se que a enzima Transaminase Glutâmico Pirúvica (TGP) encontrava-se acima da normalidade, 371 U/L.

$\mathrm{Na}$ ultrassonografia foram visualizadas anormalidades no fígado que apresentava volume aumentado, bordos regulares, parênquima homogêneo e ecogenicidade hipoecóica. Vascularização muito aumentada, sendo a imagem sugestiva de hepatopatia. Os demais órgãos observados, baço, estômago, alças intestinais, vesícula urinária e rins não apresentaram nenhum tipo de alteração, apenas este último que possuía cortical com ecogenicidade aumentada.

Apesar do suporte clínico com fluidoterapia, alimentação, o animal morreu 45 dias após a sua primeira consulta, com o diagnóstico de hepatopatia. Com isso, foi indispensável à realização da necropsia em busca do diagnóstico post mortem. As alterações encontradas na necropsia foram: caquexia do animal, mucosas discretamente hipocoradas, moderada icterícia em mucosa oral e tecido subcutâneo (Figura 1A), músculos pálidos e com moderada atrofia, discreta presença de líquido em 


\section{ACTA

cavidade torácica (Figura 1B), pulmão com coloração ora brancacenta ora avermelhado, baço de coloração enegrecida, presença de cicatriz na superfície dos rins e discreto aumento de linfonodos mesentéricos. O fígado apresentava-se moderadamente congesto, levemente amarelado e com grande quantidade de formas parasitárias compatíveis com o parasito Platynosomum spp.

Fragmentos do fígado e amostras de fezes foram coletados para exames laboratoriais.
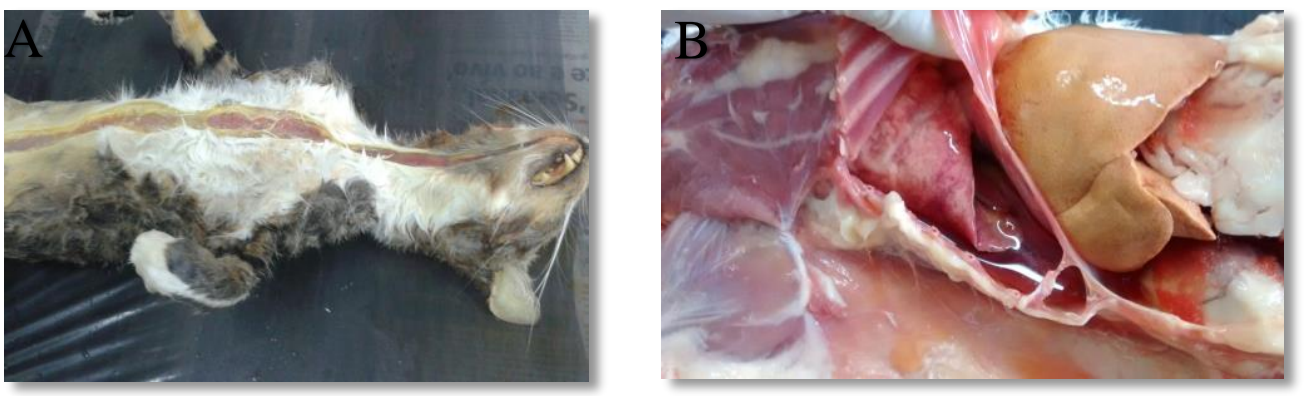

Figura 1: (A) Felino apresentando acentuada caquexia e moderada icterícia em tecido subcutâneo. (B) Presença de líquido em cavidade torácica (seta) e fígado levemente amarelado.

Os fragmentos de fígado foram enviados para o laboratório de Anatomia Patológica Veterinária da FMVV e processados pelos métodos de rotina para o exame histopatológico. Os cortes histológicos do fígado revelaram colangite crônica, com presença de hepatócitos com moderada esteatose (Figura 2 A), moderada biliestase, presença de trematódeo adulto no interior dos ductos biliares, compatível com Platynosomum spp. além de fibrose ductal e áreas de hiperplasia de ductos biliares (Figura $4 \mathrm{~B}$ e C).

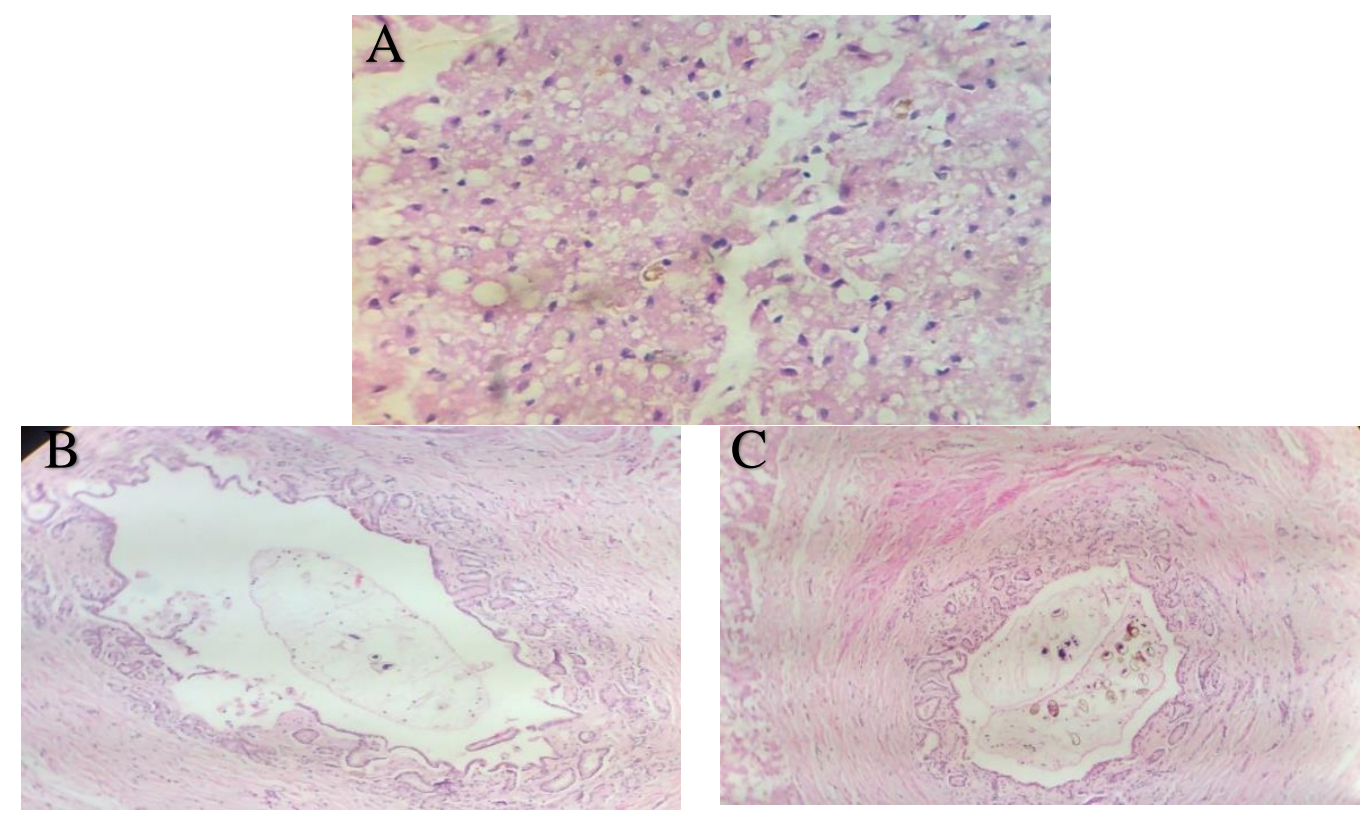

Figura 2: (A) Hepatócitos com moderada esteatose e biliestase (setas) (HE 400x). (B e C) Presença de estruturas anatômicas do trematódeo adulto no interior de ducto biliar, compatíveis ao Platynosomum spp. Observa-se ainda fibrose ao redor do ducto e hiperplasia ductal (H.E. 100x). 


\section{ACTA

As amostras de parasitos e as fezes coletadas durante a necropsia foram enviadas para o Laboratório de Parasitologia da FMVV. No exame com microscópio esterioscópico pode-se confirmar que as formas parasitárias eram compatíveis com Platynosomum spp., apresentando corpo achatado, ventosas oral e ventral, testículos dispostos lado a lado, junto e posterior à ventosa ventral, ovário deslocado da linha mediana e pós testicular (Figura 3A). O exame coproparasitológico, utilizando-se a técnica de formalina éter revelou a presença de ovos marrons, de casca espessa e operculados característico de Platynosomum spp. (Figura 3B).
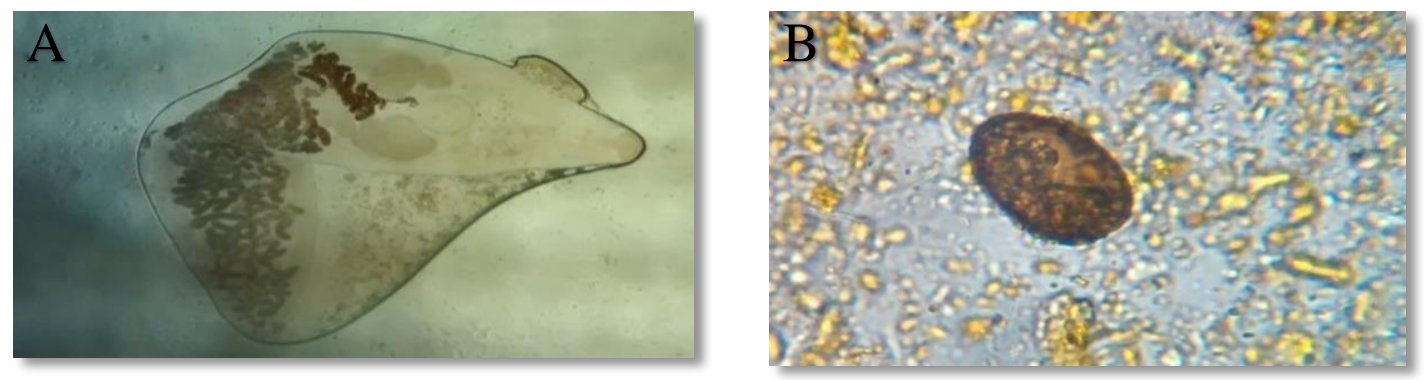

Figura 3: (A) Platynosomum spp. encontrado no fígado observado em microscópio estereoscópico. (B) Ovo de Platynosomum spp. encontrado em exame coproparasitológico, de conteúdo intestinal através da técnica de formalina

3 Discussão éter.

Inicialmente a conduta médica adotada, após exame físico, foi à realização de fluidoterapia, administração de vitaminas e implementação de sonda nasogástrica para alimentação. Estas medidas foram importantes para tentar estabilizar o animal (FILHO et al., 2015), já que este apresentava-se desidratado e sem se alimentar.

A platinosomose descrita no trabalho demonstrou inicialmente sinais inespecíficos como letargia, icterícia e desidratação, relatados também nos trabalhos de Sampaio et al. (2006) e Michaelsen et al. (2012). Tais sinais comuns a outras doenças acabam por confundir o diagnóstico da parasitose. Desta maneira é indispensável à solicitação de exames complementares para auxílio no fechamento do diagnóstico, como afirma a literatura. A ultrassonografia por exemplo, possibilita a identificação de anormalidades no fígado, principal órgão acometido pela parasitose (AZEVEDO et al., 2013). Os resultados ultrassonográficos de Sampaio et al. (2006) foram compatíveis com o do relato, onde também foram descritos que o fígado se encontrava aumentado, hipoecóico e com bordos regulares, sugestivo de hepatopatia. Os demais órgãos visualizados, bexiga, rins, baço, assim como no presente relato, também não apresentaram alterações.

Segundo Soldan et al. (2011) alterações em hemogramas de felinos parasitados por Platinossomum spp, podem sugerir a parasitose, apesar de não ser específico. No caso do exame hematológico solicitado pela Policlínica para a gata com platinossomose, não houve alterações que a evidenciassem, reafirmando Soldan et al. (2011) que em seu trabalho elucida que modificações no exame de sangue não são patognomônicas para a parasitose.

A avaliação da bioquímica sérica em casos de platinosomose direciona o médico veterinário a uma possível lesão hepática pelo aumento da TGP e de outras enzimas. A TGP encontra-se presente no fígado, livre no citoplasma, dentro de mitocôndrias, e também em boa quantidade nos músculos e hemácias, o que não a torna tão específica para determinação de lesão no fígado, desta forma deve-se associar a outros parâmetros para fechamento do diagnóstico de problema hepático (NORMAN, 2014). 


\section{ACTA

A realização da necropsia é muito importante tanto para animais diagnosticados ante mortem, quanto para os não foram diagnosticados. Sampaio et al. (2006), durante o exame post mortem em um felino com a parasitose encontraram vísceras abdominais com icterícia, hepatomegalia, fibrose e aumento de canalículos biliares, ducto e vesícula biliar. No relato de caso de Terra et al. (2015), o fígado apresentava consistência aumentada e padrão lobular acentuado. Filho et al. (2015), ao realizarem cortes histológicos no fígado verificaram colangite crônica, hepatócitos com esteatose e hiperplasia ductal, assim como no felino do presente estudo.

Santos et al. (1981), em seu trabalho verificaram a presença do parasito no interior dos ductos biliares, o que promoveu espessamento dos ductos e fibrose dos mesmos. Ferreira et al. (1999) confirmou que a evolução da doença é realmente crônica, produzindo aspecto lobular com infiltração inflamatória e congestão discreta. Tal inflamação crônica associada à obstrução pode levar a cirrose, modificando assim o tecido original do fígado por fibroso, o que leva o órgão a perder sua função, ou ficar reduzida (MONTSERIN et al., 2013).

A coleta de material fecal ante ou post mortem de um animal é válido para identificação de parasitoses variadas. No caso de platinosomose o diagnóstico definitivo é dado por meio de exame coproparasitológico, com achados de ovos operculados nas fezes caso os parasitos não tenham obstruído o ducto biliar totalmente (MARTINS, 2012). Tal exame foi realizado a partir da coleta de conteúdo fecal na necropsia, direto das alças intestinais. Na visualização em microscópio foram observados ovos amarronzados e operculados, assim como o descrito por Bielsa e Greiner (1985) e por Castro e Albuquerque (2008), corroborando assim a platinosomose.

A eficiência do tratamento adotado para platinosomose depende do grau e da extensão dos danos causados pelo parasito no animal. O fármaco praziquantel é o antiparasitário mais usado, com bons índices de cura (SOLDAN et al., 2011). A dose administrada por Moraes et al. (2015) em seu trabalho foi de $30 \mathrm{mg} / \mathrm{kg}$, por via oral, uma vez ao dia, durante cinco dias consecutivos, tendo bons resultados com a eliminação do parasitismo. No entanto, quanto mais precoce for o diagnóstico, maiores são as chances de cura e sobrevivência do felino. O uso de terapia suporte é sempre preconizado em animais parasitados (PAULA, 2010).

\section{Conclusão}

A platinosomose é uma parasitose comum que causa vários prejuízos à saúde do felino, podendo levá-lo a morte. Como os sinais clínicos são inespecíficos é indispensável à solicitação de exames complementares como o coproparasitológico e ultrassonografia, na tentativa de se realizar o diagnóstico precoce. $\mathrm{O}$ exame anatomohistopatológicoé de suma importância para o diagnóstico conclusivo de platinossomose.

\section{Referências}

AZEVEDO, F. D. et al. Avaliação radiográfica e ultrassonográfica do fígado e da vesícula biliar em gatos domésticos (Felis catus domesticus) parasitados por Platynosomum illiciens (BRAUN, 1901) Kossak, 1910. Revista Brasileira de Medicina Veterinária, v. 35, n. 3, p. 283-288, jul/set 2013.

BIELSA, L. M.; GREINER, E. C. Liver Flukes (Platynosomum concinnum in Cats). Journal of the American Hospital Association, v. 21, p. 269-274, mar/ abr 1985. 
CARREIRA, V. S.et al. Feline cholangitis/cholangiohepatitis complex secondary to Platynosomum fastosum infection in a cat. Revista Brasileira de Parasitologia Veterinária, v. 17, suplemento 1, p. 184-187, 2008.

CASTRO, L. S.; ALBUQUERQUE, G. R. Ocorrência de Platynosomum illiciens em felinos selvagens mantidos em cativeiro no Estado da Bahia, Brasil. Revista Brasileira de Parasitologia Veterinária, v. 17, n. 4, p. 239-241, 2008.

FERREIRA, A. M. R.; ALMEIDA, E. C. P. Liver Fluke Infection (Platynosomum concinnum) in Brazilian cats: Prevalence and Pathology. FelinePratice, v. 27, n. 2, p. 19-22, 1999.

FILHO, R. P. S. et al.Primeiro relato de infecção natural pelo Platynosomum spp. em gato doméstico no município de Fortaleza, Ceará, Brasil.Arquivos de Ciências Veterinárias e Zoologia da UNIPAR, v. 18, n. 1, p. 59-63, jan./mar. 2015.

FRANCO, P. A. et al. Pesquisa de Platynosomum Concinnum por técnica de sedimentação em felinos domésticos. In 34 Congresso Brasileiro da Associação Nacional de Clínicos Veterinários de Pequenos Animais, maio 2013; Anais. Natal: Anclivepa - RN 2013, p. 287 - 289.

LEAL, P. D. S. A. et al. Avaliação da administração oral de ácido ursodesoxicólico (audc) no diagnóstico da infeçção natural por Platynosomum illiciens em gatos. Revista Brasileira de Medicina Veterinária, v. 33, n. 4, p. 229-233, out/dez. 2011.

MARTINS, S. B. U. Diagnósticos diferenciais de icterícia em pacientes felinos. 2012. 45f. Trabalho monográfico (Curso de Pós Graduação "Lato Sensu" em Clínica Médica e Cirurgia de Felinos) Universidade Paulista, Belo Horizonte, 2012.

MICHAELSEN, R. et al. Platynosomum concinnum (Trematoda: Dicrocoeliidae) em gato doméstico da cidade de Porto Alegre, Rio Grande do Sul, Brasil. Veterinária em Foco, v. 10, n. 1, p. 53-60, 2012. Disponível em: <http://www.periodicos.ulbra.br/index.php/veterinaria/article/view/1167>. Acesso em: 22 mai. 2016.

MONTSERIN, S. A. S.et al. Clinical case: Platynosomum fastosum Kossack, 1910 infection in a cat: First reported case in Trinidad and Tobago. Revue Médecine Vétérinaire, v. 164, n. 1, p. 9-12, 2013. Disponível em: <http://www.revmedvet.com/search_fr.php>. Acesso em: 31 mai. 2016.

MORAES, A. V. et al. Relato do primeiro diagnóstico parasitológico de Platynosomum, Looss (1907) em felino no Estado de Santa Catarina. 2015. 5f. Trabalho da Mostra Nacional de Iniciação Científica e Tecnológica e Interdisciplinar -Instituto Federal Catarinese - 2015.

NORMAN, P. S. H. Diagnóstico diferencial de doenças hepatobiliares em gatos. 2014. 33f. Trabalho monográfico (Graduação) - Universidade Federal do Rio Grande Faculdade de Veterinária, Porto Alegre, RS, 2014. 
PAULA, C. L. Platinosomíase em felinos domésticos: um diferencial para obstrução biliar. 2010. 21f. Trabalho de conclusão de curso (Graduação) -Faculdade de Medicina Veterinária e Zootecnia da Universidade Estadual Paulista, Botucatu, SP, 2010.

SAMPAIO, M. A. S. et al. Infecção natural pelo Platynosomum Looss 1907, em gato no município de Salvador, Bahia. Revista Brasileira de Saúde e Produção Animal, v. 7, n. 1, p. 01-06, 2006.

SANTOS, J. A. et al. Colangiocarcinomas em gatos com parasitismo de dutos biliares por Platynosomum fastosum. Pesquisa Veterinária Brasileira, v. 1, n. 1, p. 31-36, 1981.

SOLDAN, M. H.; MARQUES, S. M. T. Platinosomose: abordagem na clínica felina. Revista da FZVA, v. $18, \quad$ n. $1, \quad 2011 . \quad$ Disponível em: $<$ http://revistaseletronicas.pucrs.br/ojs/index.php/fzva/article/viewArticle/7956>. Acesso em: 20 mar. 2016.

TERRA, J. P. et al. Diagnóstico Anatomopatológico de Linfoma Mediastínico, Aelurostrongilose e Platinosomose em um Felino. Enciclopédia Biosfera, Centro Científico Conhecer, v. 11, n. 21, p. 2167, 2015. 